\title{
GENERALIZED MAASS WAVE FORMS
}

\author{
T. MÜHLENBRUCH AND W. RAJI
}

(Communicated by Ken Ono)

\begin{abstract}
We initiate the study of generalized Maass wave forms, those Maass wave forms for which the multiplier system is not necessarily unitary. We then prove some basic theorems inherited from the classical theory of modular forms with a generalization of some examples from the classical theory of Maass forms.
\end{abstract}

\section{INTRODUCTION}

The space of generalized modular forms of integer weights arises naturally in rational conformal field theory or the theory of vertex operator algebras $[8,26$. Those are meromorphic functions defined on the upper half-plane that satisfy the transformation law under subgroups of finite index in the full modular group the same as classical modular forms, with a difference that the group multiplier appearing in the transformation law does not necessarily have absolute value one.

On the other hand, Maass wave forms are real analytic functions invariant under the action of subgroups of the full modular group, are eigenfunctions of the Laplacian operator and at most grow like polynomials at the cusps. Maass wave forms connect to several areas such as $L$-series [19, representation theory [1, 6] and other connections to Artin billiard and associated transfer operators 21.

In this paper, we initiate the study of generalized Maass wave forms, give basic properties and definitions and extend some theorems from the theory of classical modular forms. We shall show that we can construct Maass wave forms from generalized modular forms analogous to the construction of Maass wave forms from classical modular forms. We also construct Eisenstein series and Poincaré series associated to generalized Maass wave forms. We continue to study the analytic properties of the mentioned forms and in particular the Whittaker-Fourier expansion and the Maass operators.

In Lemma 2.5. we show that if we have a generalized Maass wave form on a subgroup of the full modular group and this same form is a classical Maass wave form of a smaller group contained in the subgroup, then the generalized Maass wave form is classical on the bigger group.

We then introduce vector-valued Maass wave forms that might help in establishing the Eichler cohomology of generalized Maass wave forms in future work. Going to the vector-valued case creates an easier tool to deal with integral transforms associated to the periods of Eichler integrals.

Received by the editors August 10, 2011.

2010 Mathematics Subject Classification. Primary 11F99.

Key words and phrases. Generalized Maass waveforms, generalized modular forms, vectorvalued modular forms. 


\section{Generalized MaAss Wave forms}

2.1. Preliminaries. Let $\operatorname{SL}(2, \mathbb{R})$ denote the group of $2 \times 2$ matrices with real entries and determinant 1 . The subgroup $\operatorname{SL}(2, \mathbb{Z}) \subset \mathrm{SL}(2, \mathbb{R})$ denotes the full modular group, that is, the subgroup of matrices with integer entries. It is generated by

$$
S=\left(\begin{array}{cc}
0 & -1 \\
1 & 0
\end{array}\right) \quad \text { and } \quad T=\left(\begin{array}{ll}
1 & 1 \\
0 & 1
\end{array}\right) \quad \text { satisfying } \quad S^{2}=(S T)^{3}=\mathbf{- 1},
$$

where $\mathbf{- 1} \in \mathrm{SL}(2, \mathbb{Z})$ and $\mathbf{1}$ denotes the identity matrix. The group $\operatorname{SL}(2, \mathbb{R})$ acts on the upper half-plane $\mathbb{H}=\{z \in \mathbb{C} \mid \operatorname{Im}(z)>0\}$ and its boundary $\mathbb{P}_{\mathbb{R}}=\mathbb{R} \cup\{\infty\}$ by fractional linear transformations

$$
\left(\begin{array}{ll}
a & b \\
c & d
\end{array}\right) z:= \begin{cases}\frac{a}{c} & \text { if } z=\infty \\
\infty & \text { if } z=-\frac{d}{c} \text { with } c \neq 0, \text { and } \\
\frac{a z+b}{c z+d} & \text { otherwise. }\end{cases}
$$

Moreover, we have

$$
\operatorname{Im}(\gamma z)=\frac{\operatorname{Im}(z)}{|c z+d|^{2}} \quad \text { and } \quad \frac{\mathrm{d}}{\mathrm{d} z} \gamma z=\frac{1}{(c z+d)^{2}}
$$

for all $\gamma=\left(\begin{array}{ll}a & b \\ c & d\end{array}\right) \in \mathrm{SL}(2, \mathbb{R})$.

Let $\Gamma \subset \mathrm{SL}(2, \mathbb{Z})$ be the subgroup of the full modular group with finite index. It is known that the fundamental domain $\mathcal{F}=\mathcal{F}_{\Gamma}$ of $\Gamma$ in $\mathbb{H}$ is a hyperbolic polygon containing finitely many inequivalent parabolic cusps $q_{1}, \ldots, q_{t}, t \geq 1$. We denote the set of inequivalent cusps by $\mathcal{C}=\mathcal{C}_{\Gamma}:=\left\{q_{1}, \ldots, q_{t}\right\}$.

To each cusp $q \in \mathcal{C}_{\Gamma}$ we denote the stabilizer of $q$ by $\Gamma_{q}=\left\langle\gamma_{q},-\mathbf{1}\right\rangle$. There exists a scaling matrix $g_{q} \in \mathrm{SL}(2, \mathbb{Z})$ such that

$$
q=g_{q} i \infty \quad \text { and } \quad g_{q}^{-1} \gamma_{q} g_{q}=\left(\begin{array}{cc}
1 & l_{q} \\
0 & 1
\end{array}\right)=T^{l_{q}}
$$

where $l_{q} \in \mathbb{N}$ is the width of the cusp $q$; see [11, (2.1), page 40], or [12, page 5].

A multiplier or multiplier system $v$ compatible with (complex) weight $k$ is a function

$$
v: \Gamma \rightarrow \mathbb{C}_{\neq 0}
$$

such that

$$
f(\gamma z)=v(\gamma) e^{i k \arg (c z+d)} f(z)
$$

allows non-zero solutions $f$. We call a multiplier system $v$ weakly parabolic if

$$
|v(\gamma)|=1 \quad \text { for all parabolic } \gamma \in \Gamma,
$$

as in [17, Equation (5)].

Remark 2.1. We use the convention

$$
w^{k}=|w|^{k} e^{i k \arg (w)}
$$

with $\arg (z) \in(-\pi, \pi]$ for all $z \in \mathbb{C}_{\neq 0}$ to determine the $k^{\text {th }}$ power in (2.6). 
Remark 2.2. Condition (2.6) implies in particular that $v$ satisfies the relation

$$
v(\gamma \delta) e^{i k \arg \left(c_{\gamma \delta} z+d_{\gamma \delta}\right)}=v(\gamma) v(\delta) e^{i k \arg \left(c_{\gamma}(\delta z)+d_{\gamma}\right)} e^{i k \arg \left(c_{\delta} z+d_{\delta}\right)}
$$

for all $\gamma, \delta \in \Gamma$ and $z \in \mathbb{H}$. In particular (2.8) implies that

$$
v(-\mathbf{1})=e^{-i k} \quad(\text { if }-\mathbf{1} \in \Gamma) .
$$

We also introduce the slash-action as notation. For $\gamma=\left(\begin{array}{ll}a & b \\ c & d\end{array}\right) \in \operatorname{SL}(2, \mathbb{R})$, let $k \in \mathbb{C}$ and $f$ be a function on $\mathbb{H}$. We define

$$
\left(\left.f\right|_{k} \gamma\right)(z):=e^{-i k \arg (c z+d)} f(\gamma z) \quad \text { for all } z \in \mathbb{H} .
$$

For example, (2.6) reads as $\left.f\right|_{k} \gamma=v(\gamma) f$.

2.2. Classical and generalized Maass wave forms. We briefly recall Maass wave forms.

Definition 2.3. Let $\Gamma \subset \mathrm{SL}(2, \mathbb{Z})$ be a subgroup with finite index and $v: \Gamma \rightarrow \mathbb{C}_{\neq 0}$ a unitary multiplier system compatible with the real weight $k$. A classical Maass wave form of weight $k$, multiplier $v$ for the group $\Gamma$ is a real-analytic function $u: \mathbb{H} \rightarrow \mathbb{C}$ satisfying

(1) $\left.u\right|_{k} \gamma=v(\gamma) u$ for all $\gamma \in \Gamma$,

(2) $u$ is an eigenfunction of the Laplace operator $\Delta_{k}$ with eigenvalue $\lambda \in \mathbb{R}$, i.e., $\Delta_{k} u=\lambda u$ with $z=x+i y \in \mathbb{H}$ and

$$
\Delta_{k}=-y^{2}\left(\partial_{x}^{2}+\partial_{y}^{2}\right)+i k y \partial_{x}
$$

(3) $u$ satisfies the growth condition $u\left(g_{q} z\right)=\mathcal{O}\left(y^{c}\right)$ at each cusp $q \in \mathcal{C}_{\Gamma}$ as $y \rightarrow \infty$ for some $c \in \mathbb{R}$ with $g_{q}$ being the associated scaling matrix in (2.4).

We denote the space of classical Maass wave forms by $c M(\Gamma, k, v, \lambda)$.

$u$ is called a classical Maass cusp form if $u$ satisfies the stronger growth condition $u\left(g_{q} z\right)=\mathcal{O}\left(y^{c}\right)$ at each cusp $q \in \mathcal{C}_{\Gamma}$ as $y \rightarrow \infty$ for all $c \in \mathbb{R}$.

Maass has proved in [19, Theorem 28] that the space $c M(\Gamma, k, v, \lambda)$ of Maass wave forms is finite dimensional.

Generalized Maass wave forms still keep essentially the properties 1 and 2 of Definition 2.3. However we remove the condition that the multiplier system is unitary and we weaken the growth condition. This leads to the following.

Definition 2.4. Let $\Gamma \subset \mathrm{SL}(2, \mathbb{Z})$ be a subgroup with finite index and $v: \Gamma \rightarrow \mathbb{C}_{\neq 0}$ a multiplier system compatible with the complex weight $k$. A generalized Maass wave form of weight $k$, multiplier $v$ for the group $\Gamma$ is a real-analytic function $u: \mathbb{H} \rightarrow \mathbb{C}$ satisfying

(1) $\left.u\right|_{k} \gamma=v(\gamma) u$ for all $\gamma \in \Gamma$,

(2) $u$ is an eigenfunction of $\Delta_{k}$ with eigenvalue $\lambda \in \mathbb{C}$, i.e., $\Delta_{k} u=\lambda u$,

(3) $u$ satisfies the growth condition $u\left(g_{q} z\right)=\mathcal{O}\left(e^{c y}\right)$ in each cusp $q \in \mathcal{C}_{\Gamma}$ as $y \rightarrow \infty$ for some $c \in \mathbb{R}$ with $g_{q}$ given in (2.4).

We denote the space of generalized Maass wave forms by $g M(\Gamma, k, v, \lambda)$. 
2.3. A basic lemma. Similar to [12, Lemma 3] we have the following result.

Lemma 2.5. Suppose $u: \mathbb{H} \rightarrow \mathbb{C}$ is a classical Maass wave form with respect to $(\Gamma, k, v, \lambda)$, with $\Gamma$ of finite index in $\mathrm{SL}(2, \mathbb{Z})$. (That means that $k \in \mathbb{R}$ and $|v(\gamma)|=1$ for all $\gamma \in \Gamma$.) Suppose further that $u$ is a generalized Maass wave form with respect to $\left(\Gamma^{\star}, k, v^{\star}, \lambda\right)$, where $\Gamma \subset \Gamma^{\star} \subset \mathrm{SL}(2, \mathbb{Z})$. (Note that $v^{\star}=v$ on $\Gamma$.)

Then $u$ is already a classical Maass form with respect to $\left(\Gamma^{\star}, k, v^{\star}, \lambda\right)$. That is to say: $k \in \mathbb{R}$ and $\left|v^{\star}(\gamma)\right|=1$ for all $\gamma \in \Gamma$ implies that $\left|v^{\star}(\gamma)\right|=1$ for all $\gamma \in \Gamma^{\star}$.

We adapt the proof of [12, Lemma 3] to our situation.

Proof. From the consistency condition (2.8) and the fact that $\Gamma$ has finite index in $\Gamma^{\star}$, it follows easily that $v$ assumes only finitely many distinct values on $\Gamma^{\star}$. On the other hand, if there were $\gamma^{\star} \in \Gamma^{\star}$ such that $\left|v\left(\gamma^{\star}\right)\right| \neq 1$, then by (2.8) the set $\left\{\left|v^{\star}\left(\left(\gamma^{\star}\right)^{n}\right)\right|: n \in \mathbb{Z}\right\}$ would contain infinitely many distinct values for $\left|v^{\star}\right|$ on $\Gamma^{\star}$.

We still have to check that the growth conditions of $u$ are in fact as in Definition 2.3 (3). Since $\Gamma \subset \Gamma^{\star}$ the set of cusps satisfy $\mathcal{C}_{\Gamma^{\star}} \subset \mathcal{C}_{\Gamma}$. The assumptions of the lemma imply that $u$ satisfies the stronger growth condition in Definition 2.3 in each cusp of $\mathcal{C}_{\Gamma^{\star}}$. The additional cusps in $\mathcal{C}_{\Gamma} \backslash \mathcal{C}_{\Gamma^{\star}}$ can be transformed into a cusp in $\mathcal{C}_{\Gamma^{\star}}$ by an element in $\Gamma^{\star}$. (These additional cusps are $\Gamma^{\star}$-equivalent to cusps in $\mathcal{C}_{\Gamma^{\star}}$.) Hence the stronger growth condition of Definition 2.3 is also valid for these cusps.

Therefore $u$ is a classical Maass wave form since it satisfies Definition 2.3.

\section{Some examples}

3.1. Maass wave forms. We consider Maass wave forms as for example as introduced in [11. These are real-analytic functions $u: \mathbb{H} \rightarrow \mathbb{C}$ which satisfy

(1) $u(g z)=u(z)$ for all $g \in \Gamma$ and $z \in \mathbb{H}$,

(2) $\Delta_{0} u=\lambda u$, with non-negative real eigenvalue $\lambda$, and

(3) $u\left(g_{q} z\right)=\mathcal{O}\left(y^{c}\right)$ in each cusp $q \in \mathcal{C}_{\Gamma}$ as $y=\operatorname{Im}(z) \rightarrow \infty$ for some $c \in \mathbb{R}$.

Maass wave forms are obviously also generalized Maass wave forms for weight 0 and trivial multiplier.

Maass wave forms with real weight, as considered in [4] and [22], are also generalized Maass wave forms for real weight and unitary multiplier.

3.2. Generalized modular forms. Generalized modular forms were introduced a few years back. Following 12, a generalized modular form $F$ is a holomorphic function $F: \mathbb{H} \rightarrow \mathbb{C}$ with a left finite Fourier expansion

$$
F(z)=\sum_{n=-m}^{\infty} a_{n} e^{2 \pi i n z}
$$

at each cusp and it satisfies the transformation property

$$
(c z+d)^{-k} F(\gamma z)=v(\gamma) F(z) \quad \text { for all } \gamma=\left(\begin{array}{cc}
\star & \star \\
c & d
\end{array}\right) \in \Gamma
$$

see [12, $\S 2$, Definition]. Taking

$$
u(z):=\operatorname{Im}(z)^{\frac{k}{2}} F(z),
$$

$F$ induces a generalized Maass wave form $u \in g M\left(\Gamma, k, \rho, \frac{k}{2}\left(1-\frac{k}{2}\right)\right)$. 
Indeed we have

$$
\begin{aligned}
u(\gamma z) & =\operatorname{Im}(\gamma z)^{\frac{k}{2}} F(\gamma z)=\left(\frac{\operatorname{Im}(z)}{|c z+d|^{2}}\right)^{\frac{k}{2}} F(\gamma z) \quad \text { using (2.3) } \\
& =\left(\frac{\operatorname{Im}(z)}{|c z+d|^{2}}\right)^{\frac{k}{2}}(c z+d)^{k} v(\gamma) F(z) \quad \text { using (3.2) } \\
& =\left(\frac{(c z+d)}{|c z+d|}\right)^{k} v(\gamma) \operatorname{Im}(z)^{\frac{k}{2}} F(z) \\
& =e^{i k \arg (c z+d)} v(\gamma) u(z) \quad \text { using } \frac{z}{|z|}=e^{i \arg (z)}
\end{aligned}
$$

for each $\gamma \in \Gamma$ and

$$
\begin{aligned}
\Delta_{k} u(z) & =\left(-\frac{1}{4} \mathrm{E}_{k-2}^{+} \mathrm{E}_{k}^{-}-\frac{k(k-2)}{4}\right)\left(\operatorname{Im}(z)^{\frac{k}{2}} F(z)\right) \\
& =0-\frac{k(k-2)}{4}\left(\operatorname{Im}(z)^{\frac{k}{2}} F(z)\right)=\frac{k}{2}\left(1-\frac{k}{2}\right) u(z)
\end{aligned}
$$

using (4.3), and the property that $u$ in (3.3) lies in the kernel of $\mathrm{E}_{k}^{-}$:

$$
\begin{aligned}
\mathrm{E}_{k}^{-}\left(\operatorname{Im}(z)^{\frac{k}{2}} F(z)\right)= & \left(-4 i y \partial_{\bar{z}}-k\right)\left(\operatorname{Im}(z)^{\frac{k}{2}} F(z)\right) \quad \text { using (4.2) } \\
= & -4 i y \partial_{\bar{z}}\left(\operatorname{Im}(z)^{\frac{k}{2}} F(z)\right)-k\left(\operatorname{Im}(z)^{\frac{k}{2}} F(z)\right) \\
= & -4 i y\left(\frac{i}{2} \partial_{y} \operatorname{Im}(z)^{\frac{k}{2}}\right) F(z)-4 i y \operatorname{Im}(z)^{\frac{k}{2}}\left(\partial_{\bar{z}} F(z)\right) \\
& -k \operatorname{Im}(z)^{\frac{k}{2}} F(z) \\
= & 2 y \frac{k}{2} \operatorname{Im}(z)^{\frac{k}{2}-1} F(z)-0-k \operatorname{Im}(z)^{\frac{k}{2}} F(z)=0 .
\end{aligned}
$$

$u$ satisfies the growth property in Definition 2.4 (3) since the generalized modular form $F$ has a left finite Fourier expansion.

This generalizes the example of holomorphic modular forms in [22, page 6].

3.3. Eisenstein series and Poincaré series. We use a method of constructing generalized Maass waveforms similar to the classical construction as in [5] to twist the definition of the real analytic Eisenstein series and Poincaré series by introducing a non-unitary multiplier systetem inside the sum. However, this construction will definitely affect the convergence of the series.

Definition 3.1. Let $v$ be a multiplier system for $\Gamma$ which is compatible to weight $k$. For $F: \mathbb{H} \rightarrow \mathbb{C}$ an eigenfunction of $\Delta_{k}$ define formally the generalized Poincaré Series $P(z)$ by the formal series

$$
\begin{aligned}
P(z) & =\sum_{\gamma \in \Gamma_{\infty} \backslash \Gamma} v(\gamma)^{-1}\left(\left.F\right|_{k} \gamma\right)(z) \\
& =\sum_{\gamma \in \Gamma_{\infty} \backslash \Gamma} v(\gamma)^{-1} e^{-i k \arg \left(c_{\gamma} z+d_{\gamma}\right)} F(\gamma z),
\end{aligned}
$$

where $\gamma=\left(\begin{array}{cc}\dot{c_{\gamma}} & \dot{d_{\gamma}}\end{array}\right)$ runs to a complete set of coset representatives for $\Gamma_{\infty} \backslash \Gamma$. 
It is a straightforward calculation to show that $P$ formally satisfies properties (11) and (2) of Definition 2.4 provided that the series converges absolutely. Here, $|v|$ is not necessarily 1 .

Assume for the moment that

$$
F(z)=\operatorname{Im}(z)^{\frac{k}{2}} h(z)
$$

with $h: \mathbb{H} \rightarrow \mathbb{C}$ a bounded holomorphic function. Recall from [15, Lemma 6] that

$$
|v(\gamma)| \leq K \mu(\gamma)^{\alpha}
$$

where $K$ is a positive constant and $\alpha$ is another constant depending on the modulus of the multiplier system at the generators of $\Gamma$ and $\mu(\gamma)=a^{2}+b^{2}+c^{2}+d^{2}$, where $a, b, c, d$ are the entries of $\gamma$. Recall also that there exists a constant $K_{1}$ such that

$$
\mu(\gamma) \leq K_{1}\left(c^{2}+d^{2}\right)
$$

for all $\gamma \in \Gamma_{\infty} \backslash \Gamma$. Moreover, we have from [15, Lemma 4] the following inequality:

$$
c^{2}+d^{2} \leq \frac{20}{3}|c z+d|^{2} \text {. }
$$

Combining (3.7) and (3.8) explains the absolute convergence of the series in (3.4) for large $k$ with $k>2 \alpha+1$ :

$$
\begin{aligned}
P(z) & =\sum_{\gamma \in \Gamma_{\infty} \backslash \Gamma} v(\gamma)^{-1} e^{-i k \arg \left(c_{\gamma} z+d_{\gamma}\right)} \operatorname{Im}(\gamma z)^{\frac{k}{2}} h(\gamma z) \\
& =\operatorname{Im}(z)^{\frac{k}{2}} \sum_{\gamma \in \Gamma_{\infty} \backslash \Gamma} v(\gamma)^{-1} \frac{h(\gamma z)}{\left|c_{\gamma} z+d_{\gamma}\right|^{k}} .
\end{aligned}
$$

Similarly, taking

$$
F(z)=\operatorname{Im}(z)^{\frac{1}{2}+\nu}
$$

defines a generalized Eisenstein series for $\operatorname{Re}(\nu)>\max \{\alpha, 0\}$ large enough for weight 0 and a compatible multiplier $v$. The absolute convergence of the series $P$ in (3.4) follows from

$$
\begin{aligned}
P(z) & =\sum_{\gamma \in \Gamma_{\infty} \backslash \Gamma} v(\gamma)^{-1} \operatorname{Im}(\gamma z)^{\frac{1}{2}+\nu} \\
& =\operatorname{Im}(z)^{\frac{1}{2}+\nu} \sum_{\gamma \in \Gamma_{\infty} \backslash \Gamma} v(\gamma)^{-1}\left|c_{\gamma} z+d_{\gamma}\right|^{-1-2 \nu} .
\end{aligned}
$$

Remark 3.2. Using $F(z)=\tilde{M}_{\frac{k}{2}, \nu}(4 \pi i|n| \operatorname{Im}(z)) e^{2 \pi i n \operatorname{Re}(z)}$ with $\operatorname{Re}(\nu)>\max \{\alpha, 0\}$ and following the arguments in [3, §5.1] define another type of Poincaré series.

\section{MAass operators AND Fourier expansions}

4.1. Maass operators. We denote by $\mathrm{E}_{k}^{ \pm}$the differential operators

$$
\mathrm{E}_{k}^{ \pm}= \pm 2 i y \partial_{x}+2 y \partial_{y} \pm k
$$

acting on real-analytic functions. Using $\partial_{z}=\frac{1}{2} \partial_{x}-\frac{i}{2} \partial_{y}$ and $\partial_{\bar{z}}=\frac{1}{2} \partial_{x}+\frac{i}{2} \partial_{y}$ gives

$$
\mathrm{E}_{k}^{+}=4 i y \partial_{z}+k \quad \text { and } \quad \mathrm{E}_{k}^{-}=-4 i y \partial_{\bar{z}}-k .
$$

Remark 4.1. The Maass operators are named after Hans Maass. He studied operators $\mathrm{K}_{k}$ and $\Lambda_{k}$ (see e.g. [19, page 177]), which are essentially $\mathrm{E}_{k}^{ \pm}$. 
As shown for example in 4 , $\S 6.1 .4]$, the operators $\mathrm{E}_{k \mp 2}^{ \pm} \mathrm{E}_{k}^{\mp}$ are related to $\Delta_{k}$ by

$$
\Delta_{k}=-\frac{1}{4} \mathrm{E}_{k-2}^{+} \mathrm{E}_{k}^{-}-\frac{k(k-2)}{4}=-\frac{1}{4} \mathrm{E}_{k+2}^{-} \mathrm{E}_{k}^{+}-\frac{k(k+2)}{4} .
$$

Direct calculations show that the slash-action commutes with the Laplace operator

$$
\Delta_{k}\left(\left.u\right|_{k} \gamma\right)=\left.\left(\Delta_{k} u\right)\right|_{k} \gamma \quad(\gamma \in \mathrm{SL}(2, \mathbb{R})),
$$

and interacts as follows with the Maass operators:

$$
\mathrm{E}_{k}^{ \pm}\left(\left.u\right|_{k} \gamma\right)=\left.\left(\mathrm{E}_{k}^{ \pm} u\right)\right|_{k \pm 2} \gamma \quad(\gamma \in \mathrm{SL}(2, \mathbb{R}))
$$

for all $k \in \mathbb{C}$ and smooth $u: \mathbb{H} \rightarrow \mathbb{C}$.

Lemma 4.2. $\mathrm{E}_{k}^{ \pm}$map generalized Maass wave forms of weight $k$ to generalized Maass forms of weight $k \pm 2$ :

$$
\mathrm{E}_{k}^{ \pm}: g M(\Gamma, k, v, \lambda) \rightarrow g M(\Gamma, k \pm 2, v, \lambda) .
$$

Proof. Using (4.3), we get the commutation relation

$$
E_{k}^{ \pm} \Delta_{k}=\Delta_{k \pm 2} E_{k}^{ \pm}
$$

As a result, the eigenfunctions of $\Delta_{k}$ are mapped to the eigenfunctions of $\Delta_{k \pm 2}$ by $E_{k}^{ \pm}$. (4.5) shows that the group action commutes with $E_{k}^{ \pm}$, and (4.1) shows that the growth condition of the generalized Maass wave form is compatible with $E_{k}^{ \pm}$.

4.2. Whittaker functions. Recall Whittaker's normalized differential equation

$$
\frac{d^{2}}{d y^{2}} G(y)+\left(-\frac{1}{4}+\frac{k}{y}+\frac{\frac{1}{4}-\nu^{2}}{y^{2}}\right) G(y)=0
$$

for smooth functions $G:(0, \infty) \rightarrow \mathbb{C}$ and $\nu \notin-\frac{1}{2} \mathbb{N}$.

According to [7, (13.14.2), (13.14.3)] (see also [20, Chapter 7]), we have two solutions $M_{k, \nu}(y)$ and $W_{k, \nu}(y)$ with different behavior as $y \rightarrow \infty$ :

$$
\begin{aligned}
& M_{k, \nu}(y) \sim \frac{\Gamma(1+2 \nu)}{\Gamma\left(\frac{1}{2}+\nu+k\right)} e^{\frac{1}{2} y} y^{-k} \quad \text { and } \\
& W_{k, \nu}(y) \sim e^{-\frac{1}{2} y} y^{k} .
\end{aligned}
$$

The asymptotic behavior is valid for $k-\nu \notin\left\{\frac{1}{2}, \frac{3}{2}, \frac{5}{2}, \ldots\right\}$; see [7, (13.14.20), (13.14.21)]. These functions satisfy also the recurrence relations [7, (13.15.1), (13.15.11)] and differentiation relations [7, (13.15.17), (13.15.20) and (13.15.23), (13.15.26)] and [20, §7.2.1, page 302].

We consider a modified pair of solutions.

Definition 4.3. For $k-\nu \notin-\frac{1}{2}+\mathbb{N}$ we define for all $y \in(0, \infty)$ :

$$
\begin{aligned}
& \tilde{W}_{k, \nu}(y):=W_{k, \nu}(y) \quad \text { and } \\
& \tilde{M}_{k, \nu}(y):=\frac{\Gamma\left(\frac{1}{2}+\nu-k\right)}{\Gamma(1+2 \nu)} M_{k, \nu}(y) .
\end{aligned}
$$

The definition makes sense for $\nu \in-\frac{1}{2} \mathbb{N}$ since the Buchholts function

$$
\mathcal{M}_{k, \nu}: y \mapsto \frac{1}{\Gamma(1+2 \nu)} M_{k, \nu}(y), \quad y>0,
$$

remains well defined at these values of $\nu$; see [20, §7.1.1, page 297]. 
The following lemma summarizes the action of the Maass operators $\mathrm{E}_{k}^{ \pm}$on $\tilde{W}_{\frac{k}{2}, \nu}$ :

Lemma 4.4. Let $k, \nu \in \mathbb{C}$ be such that $k \pm \nu \notin \frac{1}{2}+\mathbb{Z}$ and $\lambda=\frac{1}{4}-\nu^{2}$. We have for $n>0$,

$$
\mathrm{E}_{k}^{+} \tilde{W}_{\frac{k}{2}, \nu}(4 \pi n y) e^{2 \pi i n x}=-2 \tilde{W}_{\frac{k+2}{2}, \nu}(4 \pi n y) e^{2 \pi i n x}
$$

and

$$
\mathrm{E}_{k}^{-} \tilde{W}_{\frac{k}{2}, \nu}(4 \pi n y) e^{2 \pi i n x}=\left(\frac{k(k-2)}{2}+2 \lambda\right) \tilde{W}_{\frac{k-2}{2}, \nu}(4 \pi n y) e^{2 \pi i n x} .
$$

For $n<0$ we have

$$
\mathrm{E}_{k}^{+} \tilde{W}_{-\frac{k}{2}, \nu}(4 \pi|n| y) e^{2 \pi i n x}=\left(\frac{k(k+2)}{2}+2 \lambda\right) \tilde{W}_{-\frac{k+2}{2}, \nu}(-4 \pi|n| y) e^{2 \pi i n x}
$$

and

$$
\mathrm{E}_{k}^{-} \tilde{W}_{-\frac{k}{2}, \nu}(-4 \pi|n| y) e^{2 \pi i n x}=-2 \tilde{W}_{-\frac{k-2}{2}, \nu}(-4 \pi|n| y) e^{2 \pi i n x} .
$$

Proof. See [22, proof of Lemma 4], 4, Table 4.1, page 63] or direct calculations using the recurrence formulas given in [20, $\$ 7.2 .1$, page 302].

Similar relations hold for the $\tilde{M}_{k, \nu}$-function, using formulas on [20, page 302].

Example 4.5. We compute $\mathrm{E}_{k}^{+} \tilde{M}_{\frac{k}{2}, \nu}(4 \pi n y) e^{2 \pi i n x}$ for $n>0$ :

$$
\begin{aligned}
\mathrm{E}_{k}^{+} & \tilde{M}_{\frac{k}{2}, \nu}(4 \pi n y) e^{2 \pi i n x} \\
= & \left(2 i y \partial_{x}+2 y \partial_{y}+k\right)\left(\frac{\Gamma\left(\frac{1-k}{2}+\nu\right)}{\Gamma(1+2 \nu)} M_{\frac{k}{2}, \nu}(4 \pi n y) e^{2 \pi i n x}\right) \\
= & (k-4 \pi n y) \frac{\Gamma\left(\frac{1-k}{2}+\nu\right)}{\Gamma(1+2 \nu)} M_{\frac{k}{2}, \nu}(4 \pi n y) e^{2 \pi i n x} \\
& +2 \frac{\Gamma\left(\frac{1-k}{2}+\nu\right)}{\Gamma(1+2 \nu)}\left(4 \pi n y M_{\frac{k}{2}, \nu}^{\prime}(4 \pi n y)\right) e^{2 \pi i n x} .
\end{aligned}
$$

We use an identity from [20, $\$ 7.2 .1$, page 302] to rewrite $M_{\frac{k}{2}, \nu}^{\prime}$ in (4.13):

$$
4 \pi n y M_{\frac{k}{2}, \nu}^{\prime}(4 \pi n y)=\left(\frac{1}{2}+\frac{k}{2}+\nu\right) M_{\frac{k+2}{2}, \nu}(4 \pi n y)-\left(\frac{k}{2}-\frac{4 \pi n y}{2}\right) M_{\frac{k}{2}, \nu}(4 \pi n y) .
$$

Hence we find

$$
\begin{aligned}
\mathrm{E}_{k}^{+} & \tilde{M}_{\frac{k}{2}, \nu}(4 \pi n y) e^{2 \pi i n x}=(k-4 \pi n y) \frac{\Gamma\left(\frac{1-k}{2}+\nu\right)}{\Gamma(1+2 \nu)} M_{\frac{k}{2}, \nu}(4 \pi n y) e^{2 \pi i n x} \\
& -2\left(\frac{1+k}{2}+\nu\right)\left(-\frac{1+k}{2}+\nu\right) \frac{\Gamma\left(\frac{1-(k+2)}{2}+\nu\right)}{\Gamma(1+2 \nu)} M_{\frac{k+2}{2}, \nu}(4 \pi n y) \\
& -(k-4 \pi n y) \frac{\Gamma\left(\frac{1-k}{2}+\nu\right)}{\Gamma(1+2 \nu)} M_{\frac{k}{2}, \nu}(4 \pi n y) e^{2 \pi i n x} \\
= & -2\left(\frac{1+k}{2}+\nu\right)\left(-\frac{1+k}{2}+\nu\right) \frac{\Gamma\left(\frac{1-(k+2)}{2}+\nu\right)}{\Gamma(1+2 \nu)} M_{\frac{k+2}{2}, \nu}(4 \pi n y) \\
= & \left(\frac{k(k+2)}{2}-2 \lambda\right) \tilde{M}_{\frac{k+2}{2}, \nu}(4 \pi n y) e^{2 \pi i n x} .
\end{aligned}
$$


Lemma 4.6. Let $k, \nu \in \mathbb{C}$ be such that $k \pm \nu \notin \frac{1}{2}+\mathbb{Z}$ and $\lambda=\frac{1}{4}-\nu^{2}$. We have for $n>0$,

$$
\mathrm{E}_{k}^{+} \tilde{M}_{\frac{k}{2}, \nu}(4 \pi n y) e^{2 \pi i n x}=\left(\frac{k(k+2)}{2}-2 \lambda\right) \tilde{M}_{\frac{k+2}{2}, \nu}(4 \pi n y) e^{2 \pi i n x}
$$

and

$$
\mathrm{E}_{k}^{-} \tilde{M}_{\frac{k}{2}, \nu}(4 \pi n y) e^{2 \pi i n x}=2 \tilde{M}_{\frac{k-2}{2}, \nu}(4 \pi n y) e^{2 \pi i n x} .
$$

For $n<0$ we have

$$
\mathrm{E}_{k}^{+} \tilde{M}_{-\frac{k}{2}, \nu}(4 \pi|n| y) e^{2 \pi i n x}=2 \tilde{M}_{-\frac{k+2}{2}, \nu}(-4 \pi|n| y) e^{2 \pi i n x}
$$

and

$$
\mathrm{E}_{k}^{-} \tilde{M}_{-\frac{k}{2}, \nu}(-4 \pi|n| y) e^{2 \pi i n x}=2\left(\frac{k(k-2)}{4}-\lambda\right) \tilde{M}_{-\frac{k-2}{2}, \nu}(-4 \pi|n| y) e^{2 \pi i n x} .
$$

Proof. This is proven by direct calculations using identities from [20, $§ 7.2 .1$, p. 302] to rewrite $M_{\frac{k}{2}, \nu}^{\prime}$ and the functional equation $x \Gamma(x)=\Gamma(x+1)$ of the Gamma function.

4.3. Whittaker-Fourier expansions. We consider a generalized Maass wave form $u \in g M(\Gamma, k, v, \lambda)$ with weakly parabolic multiplier $v$ and assume that we have $\lambda=\frac{1}{4}-\nu^{2}$ for some $\nu \in \mathbb{C} \backslash-\frac{1}{2} \mathbb{N}$.

Let $q \in \mathcal{C}_{\Gamma}$ be a cusp of width $l_{q}$ and $g_{q} \in \mathrm{SL}(2, \mathbb{R})$ be the associated scattering matrix; see (2.4). The action of the stabilizer $\gamma_{q}=g_{q} T^{l_{q}} g_{q}^{-1}$ implies that

$$
u_{q}:=\left.u\right|_{k} g_{q}
$$

is nearly periodic, i.e., $\left.u_{q}\right|_{k} T^{l_{q}}=v\left(\gamma_{q}\right) u_{q}$. We expect an expansion of the form

$$
u_{q}(x+i y)=\sum_{n \equiv \kappa \bmod 1} a_{n}(y) e^{\frac{2 \pi i n x}{l_{q}}}
$$

at the cusp $i \infty$, where $\kappa \in \mathbb{R}$ is given by $v\left(\gamma_{q}\right)=e^{2 \pi i \kappa}$. ( $\kappa$ is real since $v$ is a weakly parabolic multiplier.)

The coefficients $a_{n}(y)$ still depend on $y$. Since $u_{q}$ solves the partial differential equation

$$
\left(\Delta_{k}-\lambda\right) u=0
$$

if and only if

$$
\left(-y^{2} \partial_{x}^{2}-y^{2} \partial_{y}^{2}+i k y \partial_{x}-\left(\frac{1}{4}-\nu^{2}\right)\right) u(x+i y)=0
$$

we find by separation of variables that $a_{n}(y)=h\left(\frac{4 \pi \varepsilon n}{l_{q}} y\right), \varepsilon=\operatorname{sign}(n)$ and $n \neq 0$, solves the ordinary differential equation

$$
h^{\prime \prime}(t)+\left(-\frac{1}{4}+\frac{1}{2} \varepsilon k \frac{1}{t}+\frac{\frac{1}{4}-\nu^{2}}{t^{2}}\right) h(t)=0,
$$

which is the Whittaker differential equation. Solutions are the $\tilde{W}$ - and $\tilde{M}$-Whittaker functions

$$
t \mapsto \tilde{W}_{\varepsilon \frac{k}{2}, \nu}(t) \quad \text { and } \quad t \mapsto \tilde{M}_{\varepsilon \frac{k}{2}, \nu}(t)
$$


In the case $n=0$, separation of variables shows that $a_{0}(y)$ solves the ordinary differential equation

$$
t^{2} h^{\prime \prime}(t)+\left(\frac{1}{4}-\nu^{2}\right) h(t)=0
$$

its independent solutions are

$$
t \mapsto t^{\frac{1}{2}+\nu} \quad \text { and } \quad t \mapsto t^{\frac{1}{2}-\nu} .
$$

Similar to the growth condition in Definition 2.4 of generalized Maass wave forms we assume the boundary condition

$$
a_{n}(y)=\mathcal{O}\left(e^{M y}\right) \quad \text { as } y \rightarrow \infty
$$

for some $M \in \mathbb{R}$. Hence, $u_{q}$ has a Fourier-Whittaker expansion

$$
\begin{aligned}
u_{q}(x+i y)= & \sum_{\substack{n \equiv \bmod _{n \neq 0} 1 \\
n \neq 0}} A_{n}|n|^{-\frac{1}{2}} \tilde{W}_{\operatorname{sign}(n) \frac{k}{2}, \nu}\left(\frac{4 \pi|n|}{l_{q}} y\right) e^{\frac{2 \pi i n}{l_{q}} x} \\
& +\sum_{\substack{n \equiv \kappa \bmod 1 \\
n \neq 0, \frac{2 \pi|n|}{l_{q}}<M}} B_{n}|n|^{-\frac{1}{2}} \tilde{M}_{\operatorname{sign}(n) \frac{k}{2}, \nu}\left(\frac{4 \pi|n|}{l_{q}} y\right) e^{\frac{2 \pi i n}{l_{q}} x} \\
& +C_{+} y^{\frac{1}{2}+\nu}+C_{-} y^{\frac{1}{2}-\nu}
\end{aligned}
$$

for some $M \in \mathbb{R}$ (which corresponds to the constant $c$ in Definition 2.4). The $0^{\text {th }}$-term coefficients $C_{+}$and $C_{-}$vanish if $\kappa \notin \mathbb{Z}$.

The calculation above shows the following.

Proposition 4.7. Let $u \in g M(\Gamma, k, v, \lambda)$ and $q$ be a cusp in $\mathcal{C}_{\Gamma}$. Then $u\left(g_{q} z\right)$ admits a Whittaker-Fourier expansion of the form (4.21). The $0^{\text {th }}$-term vanishes if $v\left(\gamma_{q}\right) \neq 1$.

Remark 4.8. Since $\tilde{W}_{0, \nu}\left(\frac{1}{2} y\right)=\sqrt{\frac{y}{\pi}} K_{\nu}\left(\frac{1}{2} y\right)$ the expansion in (4.21) leads to the usual Fourier-Bessel expansion of classical Maass cusp forms in weight 0:

$$
u(x+i y)=\sqrt{y} \sum_{n \in \mathbb{Z}_{\neq 0}} A_{n} K_{s-\frac{1}{2}}(2 \pi|n| y) e^{2 \pi i n x} .
$$

\section{Vector-VAlued Generalized MaAss WAVE FORMS}

5.1. Induced representations. Let $G$ be a group and $H$ be a subgroup of $G$ of finite index $\mu=[G: H]$. For each representation $\chi: H \rightarrow \operatorname{End}(V)$ we consider the induced representation $\chi_{H}: G \rightarrow \operatorname{End}\left(V_{G}\right)$, where

$$
V_{G}:=\{f: G \rightarrow V ; f(h g)=\chi(h) f(g) \text { for all } g \in G, h \in H\}
$$

and

$$
\left(\chi_{H}(g) f\right)\left(g^{\prime}\right)=f\left(g^{\prime} g\right) \quad \text { for all } g, g^{\prime} \in G .
$$

For $V=\mathbb{C}$ and $\chi$ the induced representation $\chi_{H}$ is the right regular representation. (If $\chi$ is the trivial representation, $\chi(h)=1$ for all $h \in H$, then $V_{G}$ is the space of left $H$-invariant functions on $G$ or, what is the same, functions on $H \backslash G$, and the action is by right translation in the argument.) One can identify $V_{G}$ with $V^{\mu}$ using a set $\left\{\alpha_{1}, \ldots, \alpha_{\mu}\right\}$ of representatives for $H \backslash G$, i.e.,

$$
H \backslash G=\left\{H \alpha_{1}, \ldots, H \alpha_{\mu}\right\} .
$$


Then

$$
V_{G} \rightarrow V^{\mu} \quad \text { with } \quad f \mapsto\left(f\left(\alpha_{1}\right), \ldots, f\left(\alpha_{\mu}\right)\right)
$$

is a linear isomorphism which transports $\chi_{H}$ to the linear $G$-action on $V^{\mu}$ given by

$$
g \cdot\left(v_{1}, \ldots, v_{\mu}\right)=\left(\chi\left(\alpha_{1} g \alpha_{k_{1}}^{-1}\right) v_{k_{1}}, \ldots, \chi\left(\alpha_{\mu} g \alpha_{k_{\mu}}^{-1}\right) v_{k_{\mu}}\right),
$$

where $k_{j} \in\{1, \ldots, \mu\}$ is the unique index such that $H \alpha_{j} g=H \alpha_{k_{j}}$. To see this, one simply calculates

$$
\left(\chi_{H}(g) f\right)\left(\alpha_{j}\right)=f\left(\alpha_{j} g\right)=f\left(\alpha_{j} g \alpha_{k_{j}}^{-1} \alpha_{k_{j}}\right)=\chi\left(\alpha_{j} g \alpha_{k_{j}}^{-1}\right)\left(f\left(\alpha_{k_{j}}\right)\right) .
$$

In the case of the right regular representation, the identification $V_{G} \cong \mathbb{C}^{\mu}$ gives a matrix realization

$$
\chi_{H}(g)=\left(\tilde{\chi}\left(\alpha_{i} g \alpha_{j}^{-1}\right)\right)_{1 \leq i, j \leq \mu},
$$

where $\tilde{\chi}(g)=\chi(g)$ if $g \in H$ and $\tilde{\chi}(g)=0$ otherwise.

We come back to the present situation. Take $G=\mathrm{SL}(2, \mathbb{Z}), H=\Gamma, \mu=$ $[\mathrm{SL}(2, \mathbb{Z}): \Gamma]$ and $g_{1}, \ldots, g_{\mu} \in \mathrm{SL}(2, \mathbb{Z})$ as representatives of the $\Gamma$ orbits in $\mathrm{SL}(2, \mathbb{Z})$ (corresponding to the $g_{k}$ 's in (2.4) ). We start with the trivial character $\chi_{0}$ of $\Gamma$, defined as $\chi_{0}(h)=1$ if $h \in \Gamma$ and $\chi_{0}(h)=0$ if $h \notin \Gamma$. Its right regular representation is

$$
\chi_{0}(h):=\chi_{\Gamma}(h)=\left(\delta_{\Gamma}\left(g_{i} h g_{j}^{-1}\right)\right)_{1 \leq i, j \leq \mu} \quad \text { for all } h \in \mathrm{SL}(2, \mathbb{Z}) .
$$

$\chi_{0}(h)$ is is a permutation matrix for each $h$.

Let us extend the matrix representation even more.

Definition 5.1. We define the weight matrix of dimension $p$,

$$
w: \mathrm{SL}(2, \mathbb{Z}) \times \mathbb{H} \rightarrow \mathrm{GL}(p, \mathbb{C}),
$$

as a $p \times p$ matrix with complex entries satisfying

$$
w(g h, z)=w(g, h z) w(h, z)
$$

for all $z \in \mathbb{H}$ and $g, h \in \mathrm{SL}(2, \mathbb{Z})$.

Example 5.2. (1) The right regular representation $w(h, z)=\chi_{0}(h)$ of the trivial character in (5.1) is a weight matrix of dimension $\mu$.

(2) The scalar function $w(h, z)=v(h) e^{i k \arg \left(c_{h} z+d_{h}\right)}$ for $v$ a multiplier with weight $k$ for $\operatorname{SL}(2, \mathbb{Z})$ is a 1 -dimensional weight matrix.

Lemma 5.3. A multiplier $v$ of $\Gamma$ with weight $k$ induces a weight matrix $w_{k, v}$ of dimension $\mu=[\mathrm{SL}(2, \mathbb{Z}): \Gamma]$ by

$$
w_{k, v}(h, z)=\left(w_{i, j}(h, z)\right)_{1 \leq i, j \leq \mu} \quad(\text { for all } h \in \mathrm{SL}(2, \mathbb{Z}), z \in \mathbb{H})
$$

with

$$
\begin{aligned}
w_{i, j}(h, z): & = \begin{cases}v\left(g_{i} h g_{j}^{-1}\right) e^{i k \arg \left(c_{g_{i} h g_{j}} z+d_{g_{i} h g_{j}}\right)} & \text { if } g_{i} h g_{j}^{-1} \in \Gamma \text { and } \\
0 & \text { if } g_{i} h g_{j}^{-1} \notin \Gamma\end{cases} \\
& =\delta_{\Gamma}\left(g_{i} h g_{j}^{-1}\right) v\left(g_{i} h g_{j}^{-1}\right) e^{i k \arg \left(c_{g_{i} h g_{j}} z+d_{g_{i} h g_{j}}\right)}
\end{aligned}
$$

and $\delta_{\Gamma}(g)=1(=0)$ if $g \in \Gamma(\notin \Gamma)$ and $g=\left(\begin{array}{cc}\star & \star \\ c_{g} & d_{g}\end{array}\right) \in \mathrm{SL}(2, \mathbb{Z})$. 
Proof. We have to verify (5.2). Indeed, using property (2.8) we find

$$
\begin{aligned}
w_{k, v}(g h, z)= & \left(w_{i, j}(g h, z)\right)_{i, j} \\
= & \left(\delta_{\Gamma}\left(g_{i} g h g_{j}^{-1}\right) v\left(g_{i} g h g_{j}^{-1}\right) e^{i k \arg \left(c_{g_{i} g h g_{j}^{-1}} z+d_{g_{i} g h g_{j}^{-1}}\right)}\right)_{i, j} \\
= & \left(\sum_{k} \delta_{\Gamma}\left(g_{i} g g_{k}^{-1} g_{k} h g_{j}^{-1}\right) v\left(g_{i} g g_{k}^{-1} g_{k} h g_{j}^{-1}\right)\right. \\
\times & \left(\sum_{k} e^{i k \arg \left(c_{g_{i} g g_{k}^{-1} g_{k} h g_{j}^{-1}} z+d_{g_{i} g g_{k}^{-1} g_{k} h g_{j}^{-1}}\right)}\right)_{i, j} \\
& \left.\times g_{i} g g_{k}^{-1}\right) \delta_{\Gamma}\left(g_{k} h g_{j}^{-1}\right) v\left(g_{i} g g_{k}^{-1}\right) v\left(g_{k} h g_{j}^{-1}\right) \\
= & \left(\delta_{\Gamma}\left(g_{i} g g_{k}^{-1}\right) v\left(g_{i} g g_{k}^{-1}\right) e^{i k \arg \left(c_{g_{i} g g_{k}^{-1}}\left(g_{k} h g_{j}^{-1} z\right)+d_{g_{i} g g_{k}^{-1}}\right)}\right)_{i, k} \\
& \left.\times\left(\delta_{\Gamma}\left(g_{k} h g_{j}^{-1} z\right)+d_{g_{i} g g_{k}^{-1}}\right) e^{i k \arg \left(c_{g_{k} h g_{j}^{-1}} z+d_{g_{k} h g_{j}^{-1}}\right)}\right)_{i, j} \\
= & w_{k, v}(g, h z) w_{k, v}(h, z) .
\end{aligned}
$$

5.2. Vector-valued generalized Maass wave forms. Following loosely 13 and [23], we introduce vector-valued generalized Maass wave forms.

Definition 5.4. A vector-valued generalized Maass wave form (vvgMF for short) $\vec{u}: \mathbb{H} \rightarrow \mathbb{C}^{t}$ of dimension $t \in \mathbb{N}$ for $\Gamma$, weight matrix $w(\cdot, \cdot)$ and eigenvalue $\lambda \in \mathbb{C}$ is a vector-valued function $\vec{u}=\left(u_{1}, \ldots, u_{t}\right)^{\operatorname{tr}}$ satisfying

(1) $u_{j}$ is real-analytic for all $j \in\{1, \ldots, t\}$,

(2) $\vec{u}(g z)=w(g, z) \vec{u}(z)$ for all $z \in \mathbb{H}$ and $g \in \mathrm{SL}(2, \mathbb{Z})$,

(3) $\Delta_{k} u_{j}=\lambda u_{j}$ for all $j \in\{1, \ldots, t\}$ and

(4) $u_{j}(z)=\mathcal{O}\left(e^{M y}\right)$ as $\operatorname{Im}(z) \rightarrow \infty$ for all $j \in\{1, \ldots, t\}$ and some $C \in \mathbb{R}$.

The constant $M$ does not depend on $j$.

$g M_{\mathrm{vv}}(\Gamma, t, w, \lambda)$ denotes the space of all vector-valued generalized Maass wave forms.

Example 5.5. (1) Let $\vec{u}$ be a vector-valued cusp form as in [23, Definition 3.2]. Then $\vec{u}$ is also a vector-valued generalized Maass wave form, with weight matrix $w(g, z):=\rho(g)$ given in $[23,(7)]$ and dimension $t:=[\operatorname{SL}(2, \mathbb{Z}): \Gamma]$.

(2) Let $\vec{F}$ be a vector-valued modular form of weight $k$, multiplier $v$ and $p$ dimensional complex representation $\rho$ in $\operatorname{SL}(2, \mathbb{Z})$ given in [13, §1]. Then

$$
\vec{u}(z):=\operatorname{Im}(z)^{\frac{k}{2}} \vec{F}(z)
$$

defines a vector-valued generalized Maass wave form for $\operatorname{SL}(2, \mathbb{Z})$, with dimension $p$, weight matrix $w(g, z):=v(g) e^{i k \arg \left(c_{g} z+d_{g}\right)} \rho(g)$ and spectral value $\frac{k}{2}$, similar to 3.2 . 
To each $u \in g M(\Gamma, k, v, \lambda)$ we associate the vector-valued function $\Pi(u)$ given by

$$
\Pi: g M(\Gamma, k, v, \lambda) \rightarrow g M_{\mathrm{vv}}\left(\Gamma, \mu, w_{k, v}, \lambda\right) ; \quad u \mapsto \Pi(u):=\left(\left.u\right|_{k} g_{1}, \ldots,\left.u\right|_{k} g_{\mu}\right)^{\operatorname{tr}},
$$

where $\mu=[\operatorname{SL}(2, \mathbb{Z}): \Gamma]$ denotes the index of $\Gamma$. The function $\Pi(u)$ satisfies all four properties of a vector-valued generalized Maass wave form in Definition 5.4.

Indeed, take a $u \in g M(\Gamma, k, v, \lambda)$ and an $i \in\{1, \ldots, \mu\}$. Obviously, $[\Pi(u)]_{i}=$ $\left.u\right|_{k} g_{i}$ is real-analytic on $\mathbb{H}$. As mentioned in (4.4), we have

$$
\Delta_{k}[\Pi(u)]_{i}=\Delta_{k}\left(\left.u\right|_{k} g_{i}\right)=\left.\left(\Delta_{k} u\right)\right|_{k} g_{i}=\left[\Pi\left(\Delta_{k} u\right)\right]_{i} .
$$

The growth condition for $\Pi(u)$ also follows directly from the growth condition for $u$. To check the transformation property, take an $h \in \operatorname{SL}(2, \mathbb{Z})$. There exists an $h^{\prime} \in \Gamma$ and a unique $j \in\{1, \ldots, \mu\}$ such that $g_{i} h g_{j}^{-1}=: h^{\prime} \in \Gamma$. Using the transformation property of generalized Maass wave forms in Definition 2.4 we find

$$
\begin{aligned}
{[\Pi(u)]_{i}(h z) } & =u\left(g_{i} h z\right)=u\left(g_{i} h g_{j}^{-1} g_{j} z\right) \\
& =v\left(g_{i} h g_{j}^{-1}\right) e^{i k \arg \left(c_{g_{i} h g_{j}^{-1}} z+d_{g_{i} h g_{j}^{-1}}\right)} u\left(g_{j} z\right) \\
& =w_{i, j}(h, z) u\left(g_{j} z\right) \quad\left(\text { with } w_{i, j} \text { as in (5.4) }\right) \\
& \left.=\sum_{j^{\prime}=1}^{\mu} w_{i, j^{\prime}}(h, z) u\left(g_{j^{\prime}} z\right)=\left[w_{k, v}(h, z) \Pi(u)(z)\right]_{i} \quad \text { (with (5.3) }\right) .
\end{aligned}
$$

This shows the transformation property

$$
\Pi(u)(h z)=w_{k, v}(h, z) \Pi(u)(z)
$$

of vvgMFs. Hence $\Pi(u) \in g M_{\mathrm{vv}}\left(\Gamma, \mu, w_{k, v}, \lambda\right)$.

On the other hand, consider the map

$$
\pi: g M_{\mathrm{vv}}\left(\Gamma, \mu, w_{k, v}, \lambda\right) \rightarrow g M(\Gamma, k, v, \lambda), \quad \vec{u} \mapsto[\vec{u}]_{j}
$$

where $j \in\{1, \ldots, \mu\}$ satisfies $g_{j} \in \Gamma$. The map is well defined since $j$ is uniquely determined and the function $u:=\pi(\vec{u})$ is in $g M(\Gamma, k, v, \lambda)$ :

$u$ satisfies the transformation property $\left.u\right|_{k} h=v(h) u$ for all $h \in \Gamma$ since

$$
\begin{aligned}
u(h z) & =[\vec{u}(h z)]_{j}=\left[w_{k, v}(h, z) \vec{u}(z)\right]_{j} \quad(\text { transformation property of } \vec{u}) \\
& =\sum_{l=1}^{\mu} w_{j, l}(h, z)[\vec{u}(z)]_{l} \quad(\text { using }(\underline{5.3})) \\
& =w_{j, j}(h, z)[\vec{u}(z)]_{j} \quad(\operatorname{using}(\underline{5.4})) \\
& =v\left(g_{j} h g_{j}^{-1}\right) e^{i k \arg \left(c_{g_{i} h g_{j}} z+d_{g_{i} h g_{j}}\right)} u(z) \\
& =v(h) e^{i k \arg \left(c_{h} z+d_{h}\right)} u(z) \quad(\operatorname{using}(\underline{2.8})) .
\end{aligned}
$$

Obviously, $u$ is also an eigenfunction of $\Delta_{k}$ with eigenvalue $\lambda$. 
To show that $u$ satisfies the required growth condition in all cusps, take a cuspidal point $q \in \mathcal{C}_{\Gamma}$ of $\Gamma$ and $g_{q} \in \mathrm{SL}(2, \mathbb{Z})$ satisfying $q=g_{q} i \infty$, as in (2.4). Similar to the calculation above, we find

$$
\begin{aligned}
{\left[\vec{u}\left(\gamma g_{q} z\right)\right]_{j} } & =\left[w_{k, v}\left(g_{q}, z\right) \vec{u}(z)\right]_{j}=\sum_{l=1}^{\mu} w_{j, l}\left(g_{q}, z\right)[\vec{u}(z)]_{l} \quad \text { (using (5.3)) } \\
& =w_{j, q}\left(g_{q}, z\right)[\vec{u}(z)]_{q} \quad(\text { using (5.4) }),
\end{aligned}
$$

since $g_{j} g_{q} g_{k}^{-1} \notin \Gamma$ except for $k=q$. Hence

$$
\begin{aligned}
u\left(g_{q} z\right) & =w_{j, q}\left(g_{q}, z\right)[\vec{u}(z)]_{q}=v\left(g_{j}\right) e^{i k \arg \left(c_{g_{j}} z+d_{g_{j}}\right)}[\vec{u}(z)]_{q} \\
& =\mathcal{O}\left(e^{M y}\right) \quad \text { as } y \rightarrow \infty \text { for some } M \in \mathbb{R} .
\end{aligned}
$$

Lemma 5.6. - The maps $\Pi$ and $\pi$ are inverses of each other.

- The spaces $g M_{v v}\left(\Gamma, \mu, w_{k, v}, \lambda\right)$ and $g M(\Gamma, k, v, \lambda)$ are bijective.

\section{Conclusions And outlook}

In this paper, we introduced generalized Maass wave forms, which extend the generalized modular forms introduced in 12 and, simultaneously, Maass wave forms of real weight, as discussed in 44. We also proved some related theorems and discussed the expansions of those forms, which generalize the classical theory of Maass forms. On the other hand, several examples were also introduced taking into account the bound for the multiplier system.

As a next step, we would like to extend the concept of Eichler integrals leading to period polynomials [9] and period functions [18, 22 attached to modular cusp forms and Maass cusp forms. That is, we would like to generalize objects of the form

$$
(g, \gamma) \mapsto \int_{z_{0}}^{\gamma z_{0}} g(z)(z-X)^{k-2} d z
$$

where $g: \mathbb{H} \rightarrow \mathbb{C}$ is a modular cusp form of weight $k$ and $\gamma \in \Gamma$, to the setting of generalized Maass wave forms. Hence we plan to follow [16, 17] in our setting and we plan as well to characterize the cohomology group associated to those forms. In the end, we aim to construct an Eichler-Shimura-type map between the space of generalized Maass wave forms and the suitable group cohomology.

It is worth mentioning that the vector-valued Maass wave forms are introduced in this paper for computational purposes in our future work similar to the use of vector-valued Maass cusp forms in [23. Also, to allow weight matrices instead of the scalar-valued multiplier systems and weight factors seems to be an interesting generalization along [13. This way, we can easily pull back relations on forms for $\Gamma \subset \mathrm{SL}(2, \mathbb{Z})$ to matrix-valued relations on vector-valued forms for $\operatorname{SL}(2, \mathbb{Z})$ as illustrated in $[23$.

\section{ACKNOWLEDGMENTS}

The authors would like to thank the referee for an excellent comment about the algebraic aspect of these forms and the importance of pursuing this aspect. In addition, the authors would like to thank the Center for Advanced Mathematical Sciences (CAMS) at the American University of Beirut for the opportunity to meet in a workshop and discuss this work. 


\section{REFERENCES}

[1] A. Borel, Automorphic forms on $\mathrm{SL}_{2}(\mathbf{R})$. Cambridge Tracts in Mathematics 130. Cambridge University Press, Cambridge, 1997. MR.1482800 (98j:11028)

[2] R.W. Bruggeman, Fourier coefficients of cusp forms. Inventiones Mathematicae 45 (1978), 1-18. MR0472701 (57:12394)

[3] R.W. Bruggeman, Fourier coefficients of automorphic forms. Lecture Notes in Mathematics 865. Springer-Verlag, Berlin-New York, 1981. MR.643241 (84h:10034)

[4] R.W. Bruggeman, Families of automorphic forms. Monographs in Mathematics 88, Birkhäuser, 1994. MR1306502 (95k:11060)

[5] J.H. Bruinier, K. Ono, and R.C. Rhoades, Differential operators for harmonic weak Maass forms and the vanishing of Hecke eigenvalues. Mathematische Annalen 342 (2008), 673-693. Errata. Mathematische Annalen 345 (2009), 31. MR2430995 (2009f:11046) MR2520050 (2010h:11070)

[6] D. Bump, Automorphic forms and representations. Cambridge Studies in Advanced Mathematics 55. Cambridge University Press, Cambridge, 1997. MR1431508 (97k:11080)

[7] Digital Library of Mathematical Functions. 2010-05-07. National Institute of Standards and Technology, from http://dlmf.nist.gov/.

[8] C. Dong, H. Li, G. Mason, Modular invariance of trace functions in orbifold theory. Communications in Mathematical Physics 214 (2000), 1-56. MR.1794264 (2001k:17043)

[9] M. Eichler, Eine Verallgemeinerung der Abelschen Integrale. Mathematische Zeitschrift 67 (1957), 267-298. MR0089928 (19:740a)

[10] M. Eichler, Grenzkreisgruppen und kettenbruchartige Algorithmen. Acta Arithmetica 11 (1965), 169-180. MR0228436 (37:4016)

[11] H. Iwaniec, Spectral methods of automorphic forms. Graduate Studies in Mathematics 53, American Mathematical Society, 2002. MR1942691 (2003k:11085)

[12] M. Knopp and G. Mason, Generalized modular forms. Journal of Number Theory 99 (2003), 11-28. MR 1957241 (2003k:11061)

[13] M. Knopp and G. Mason, On vector-valued modular forms and their Fourier coefficients. Acta Arithmetica 110 (2003), 117-124. MR2008079 (2004j:11043)

[14] M. Knopp and G. Mason, Vector-valued modular forms and Poincaré series. Illinois Journal of Mathematics 48 (2004), 1345-1366. MR2114161(2005k:11086)

[15] M. Knopp, Some new results on the Eichler cohomology of automorphic forms. Bulletin of the American Mathematical Society 80 (1974), 607-632. MR0344454 (49:9193)

[16] M. Knopp, J. Lehner and W. Raji, Eichler cohomology for generalized modular forms. International Journal of Number Theory 5 (2009), 1049-1059. MR.2569744 (2010i:11052)

[17] M. Knopp and W. Raji, Eichler cohomology and generalized modular forms II. International Journal of Number Theory 6 (2010), 1083-1090. MR.2679457(2011f:11066)

[18] J. Lewis and D. Zagier, Period functions for Maass wave forms. I. Annals of Mathematics, Second Series 153 (2001), 191-258. MR1826413 (2003d:11068)

[19] H. Maass, Lectures on modular functions of one complex variable. Tata Institute of Fundamental Research, second edition, 1983. MR734485 (85g:11034)

[20] W. Magnus, F. Oberhettinger and R. P. Soni, Formulas and theorems for the special functions of mathematical physics. $3^{\text {rd }}$ edition. In: Die Grundlehren der mathematischen Wissenschaften 52, Springer-Verlag, 1966. MR0232968 (38:1291)

[21] H. Mayer, The thermodynamic formalism approach to Selberg's zeta function for PSL(2, Z). Bulletin of the American Mathematical Society, New Series 25 (1991), 55-60. MR.1080004 (91j:58130)

[22] T. Mühlenbruch, Systems of automorphic forms and period functions. Ph.D. Thesis, Utrecht University, 2003.

[23] T. Mühlenbruch, Hecke operators on period functions for $\Gamma_{0}(n)$. Journal of Number Theory 118 (2006), 208-235. MR2225281 (2007i:11062)

[24] W. Raji, Construction of generalized modular integrals. Functiones et Approximatio Commentarii Mathematici 41 (2009), 105-112. MR2590327 (2010k:11078)

[25] W. Raji, Fourier coefficients of generalized modular forms of negative weight. International Journal of Number Theory 5 (2009), 153-160. MR2499027 (2010a:11071)

[26] Y. Zhu, Modular invariance of characters of vertex operator algebras. Journal of the American Mathematical Society 9 (1996), 237-302. MR1317233(96c:17042) 
Department of Mathematics and Computer Science, Fern Universität in Hagen, 58084 Hagen, Germany

E-mail address: tobias.muehlenbruch@fernuni-hagen.de

$U R L:$ http://www.fernuni-hagen.de

Department of Mathematics, American University of Beirut, Beirut, Lebanon

E-mail address: wr07@aub.edu.lb

URL: http://www .aub.edu.lb/fas/math/ 